\title{
COMMENTARY
}

\section{Complications of continuous renal replacement therapy in children: are all created equal?}

\author{
Tina L Palmieri* \\ See related research by Santiago et al., http://ccforum.com/content/13/6/R184
}

\begin{abstract}
Continuous renal replacement therapy (CRRT) in pediatric acute kidney dysfunction has evolved in recent decades; however, little objective data exist for complications associated with CRRT. Santiago and colleagues are among the first to document four complications of acute kidney dysfunction in critically ill children: catheterization-related insertion complications, hypotension, hemorrhage, and electrolyte disturbances. They reported that hypotension at connection (41.3\%) and electrolyte disturbance (50.6\%) were the leading complications. Although this study is limited by small sample size and the outcome variables measured, it is an important first step in assessing outcomes of CRRT in children. A prospective multicenter randomized trial will be needed to fully delineate the complications and define the risk/benefit ratio of CRRT in children.
\end{abstract}

The treatment of acute kidney dysfunction in critically ill children has advanced markedly in the past several decades. Currently, continuous renal replacement therapy (CRRT) and peritoneal dialysis form the mainstays of treatment. However, there is little objective information on patient outcomes and complications associated with CRRT. Santiago and colleagues [1] have taken the initiative to study CRRT complications and the factors contributing to those complications in 174 critically ill children treated from January 1996 to June 2009 at a single pediatric intensive care unit. Complications associated with catheterization, hypotension at the time of CRRT connection, hemorrhage, and electrolyte disturbance in the first 72 hours were analyzed. The authors conclude that hypotension and electrolyte disturbances are the most common CRRT complications in children.

*Correspondence: tina.palmieri@ucdmc.ucdavis.edu

University of California Davis Regional Medical Center, Shriners Hospital for Children Northern California, 2425 Stockton Blvd, Sacramento, CA 95817, USA
The treatment of acute kidney injury in children has been evolving over the past 20 years. In 1995, 45\% of pediatric centers used peritoneal dialysis and 18\% used CRRT for acute kidney injury. In 1999 that number was reversed: $31 \%$ of centers used peritoneal dialysis, while $36 \%$ used CRRT [2]. This change may be associated with the changing epidemiology of kidney injury (currently, the leading causes of acute kidney injury in the pediatric intensive care unit are a comorbidity/complication of another disease such as congenital heart disease, nephrotoxic medications, and sepsis) as opposed to primary renal disease, which was the most common cause in the 1980s $[3,4]$. The paper by Santiago and colleagues [1], identifying the complications of CRRT, is a unique and important step in determining the risk/benefit ratio of CRRT in children and providing the sorely needed information upon which to judge the appropriate use of CRRT. The data presented can serve as the foundation for a future multicenter trial assessing the utility of CRRT in children.

This study, like all good studies, has several important limitations. A relatively small (174) number of children were studied over a 13-year period at a single center, limiting its generalizability. During this time, the clinical management of critically ill children likely changed, which could be a confounding factor in the study. For example, as CRRT technology developed during the study, several different CRRT pumps were used. In addition, several different catheter sizes were used, which could impact circuit viability.

Hypotension at dialysis onset is an important complication of therapy, but could be related to multiple factors, including how the patient was connected to the circuit (either directly primed with saline or after purging with heparin and flushed with albumin). This aspect of the study is particularly unique in that hypotension with dialysis onset is clinically accepted but its incidence seldom reported. The high frequency of hypotension at dialysis onset suggests that practitioners may need to change how dialysis is started.

Another seldom-reported complication of dialysis, electrolyte abnormalities during CRRT, is also documented in this study. Unfortunately, electrolytes were 
monitored for only 72 hours, and the significance of these electrolyte abnormalities is not presented. An isolated electrolyte abnormality does not necessarily constitute a clinically significant complication. Finally, fluid balance complications are intricately linked to electrolyte abnormalities as well as to mechanical ventilation and outcomes, but no data on fluid-related complications are presented.

In summary, Santiago and colleagues have taken the important first step in evaluating the use of CRRT in critically ill children: identifying complications of therapy. This foundation study provides the baseline for future multicenter prospective studies with the appropriate statistical power to delineate fully the relative incidence and severity of complications associated with CRRT.

Abbreviations

CRRT = continuous renal replacement therapy.

Competing interests

The author declares that she has no competing interests.
Published: 26 January 2010

\section{References}

1. Santiago MJ, Lopez-Herce J, Urbano J, Solana MJ, Del Castillo JD, Ballestero Y, Botran M, Bellon JM: Complications of continuous renal replacement therapy in critically ill children: a prospective observational evaluation study. Crit Care 2009, 13:R184.

2. Warady BA, Bunchman T: Dialysis therapy for children with acute renal failure: survey results. Pediatr Nephrol 2000, 15:11-13.

3. Bunchman TE, McBryde KD, Mottes TE, Gardner JJ, Maxvold NJ, Brophy PD: Pediatric acute renal failure: outcome by modality and disease. Pediatr Nephrol 2001, 16:1067-1071.

4. Hui-Stickle S, Brewer ED, Goldstein SL: Pediatric ARF epidemiology at a tertiary care center from 1999 to 2001. Am J Kidney Dis 2005, 45:96-101.

doi:10.1186/cc8229

Cite this article as: Palmieri TL: Complications of continuous renal replacement therapy in children: are all created equal? Critical Care 2010, 14:105. 